\title{
Cost-effectiveness of obinutuzumab versus rituximab biosimilars for previously untreated follicular lymphoma
}

\author{
Scott J Spencer, MPA, MA; Gregory F Guzauskas, MSPH, PhD; Federico Felizzi, PhD, MBA; Aino Launonen, MSc; \\ Keith Dawson, DNP, MS, RN; David L Veenstra, PharmD, PhD; and Anthony Masaquel, PhD, MPH
}

\section{What is already known about this subject}

- Obinutuzumab significantly improves progression-free survival compared with rituximab when used with chemotherapy for the first-line treatment of patients with follicular lymphoma (FL).

- Pharmacoeconomic data have shown obinutuzumab-based chemoimmunotherapy to be costeffective, despite increased drug costs when compared with rituximab.

- Rituximab biosimilars are less costly relative to rituximab, which has implications for the cost-effectiveness of obinutuzumab.

\section{What this study adds}

- We have used a decision-analytic model to investigate the economic implications for health care providers of using obinutuzumab rather than rituximab biosimilars in this setting.

- We modeled costs and quality-adjusted life-years over a lifetime horizon, accounting for direct costs only, from a U.S. health care perspective.

- Our findings indicate that obinutuzumab plus chemotherapy is projected to be cost-effective versus rituximab biosimilars plus chemotherapy in the United States as first-line treatment for FL, with this cost-effectiveness due to increased quality-adjusted life-years gained and cost savings accruing from delayed disease progression.

the cost-effectiveness of $\mathrm{G}+$ chemo versus $\mathrm{Ra}+$ chemo and $\mathrm{Rp}+$ chemo has not yet been estimated.

BACKGROUND: In the randomized phase 3 GALLIUM trial, first-line treatment with obinutuzumab (GA101; G) plus chemotherapy $(\mathrm{G}+$ chemo) resulted in superior progressionfree survival (PFS) compared with rituximab plus chemotherapy $(\mathrm{R}+\mathrm{chemo})$ for patients with follicular lymphoma (FL). G + chemo was found to be cost-effective when compared with $\mathrm{R}+$ chemo (incremental cost-effectiveness ratio [ICER] of approximately $\$ 2,300$ per quality-adjusted life-year [QALY] gained). Two rituximab biosimilars, rituximab-abbs ( $\mathrm{Ra}$ ) and rituximab-pvvr (Rp), have been approved by the FDA for use in this setting. However,
OBJECTIVE: To evaluate the cost-effectiveness of $\mathrm{G}+$ chemo versus $\mathrm{Ra}+$ chemo and $\mathrm{Rp}+$ chemo in the first-line treatment of FL.

METHODS: We adapted an existing Markov model that compared $\mathrm{G}+$ chemo with $\mathrm{R}+$ chemo, using investigator-assessed PFS and postprogression survival data from the GALLIUM trial to model overall survival. All patients in the study received induction chemoimmunotherapy with either $\mathrm{G}+$ chemo or $\mathrm{R}+$ chemo, with responders then receiving obinutuzumab or rituximab maintenance therapy for 2 years or until disease

\section{Author affiliations}

Scott J Spencer, MPA, MA, University of Washington Institute for Public Health Genetics, Seattle. Gregory F Guzauskas, $\mathrm{MSPH}, \mathrm{PhD}$, and David L Veenstra, PharmD, $\mathrm{PhD}$, University of Washington Department of Pharmacy, Seattle. Federico Felizzi, $\mathrm{PhD}, \mathrm{MBA}$, and Aino Launonen, MSc, F. Hoffmann-La Roche, Basel, Switzerland. Keith Dawson, DNP, MS, RN, and Anthony Masaquel, PhD, MPH, Genentech, South San Francisco, CA.

AUTHOR CORRESPONDENCE:

David L Veenstra, veenstra@uw.edu

J Manag Care Spec Pharm. 2021;27(5):615-24

Copyright $(2021$, Academy of Managed Care Pharmacy. All rights reserved.

progression. We assumed that the efficacy and safety of the rituximab biosimilars plus chemotherapy were the same as the $\mathrm{R}+$ chemo arm of the GALLIUM study. Drug utilization and treatment duration were also derived from GALLIUM. Health care costs were based on Medicare reimbursements, and drug costs were average sale prices for intravenous therapies or wholesale acquisition costs for oral therapies used after progression. Utility estimates were based on the GALLIUM trial data and published literature. Sensitivity analyses were conducted to assess the key drivers of the model and uncertainty in the results. 
Results: Treatment with G + chemo led to an increase of 0.93 QALYs relative to rituximab biosimilars plus chemotherapy ( $95 \%$ credible range $[C R]=0.36-1.46$ ). The total cost of $\mathrm{G}+$ chemo was $\$ 191,317$, whereas the total costs of $\mathrm{Ra}+$ chemo and $\mathrm{Rp}+$ chemo were $\$ 164,340$ $(\triangle 14.1 \%)$ and $\$ 169,755(\triangle 11.3 \%)$, respectively, with $\mathrm{G}+$ chemo resulting in incremental costs of $\$ 26,978(95 \% C R=\$ 19,781-\$ 33,119)$ and $\$ 21,562$ (95\% CR $=\$ 14,473-\$ 28,389)$, respectively. The incremental total drug and administration costs were $\$ 32,678(\Delta 25.4 \%)$ and $\$ 27,263(\Delta 21.2 \%)$ for $\mathrm{G}+$ chemo versus $\mathrm{Ra}+$ chemo and $\mathrm{G}+$ chemo versus $\mathrm{Rp}+$ chemo, respectively. There were cost savings of $\$ 7,050$ $(\triangle-12.4 \%)$ related to disease progression for $\mathrm{G}+\mathrm{chemo}(\$ 56,727)$ compared with $\mathrm{Ra}+$ chemo and $\mathrm{Rp}+$ chemo $(\$ 63,777)$. ICERs were $\$ 28,879$ and $\$ 23,082$ per QALY gained for $\mathrm{G}+$ chemo versus $\mathrm{Ra}+$ chemo and $\mathrm{Rp}+$ chemo, respectively. In probabilistic sensitivity analyses, $\mathrm{G}+$ chemo was cost-effective at the $\$ 50,000$ and $\$ 100,000$ per QALY thresholds versus both $\mathrm{Ra}+$ chemo ( $88 \%$ and $98 \%$ probabilities of cost-effectiveness, respectively) and Rp + chemo ( $93 \%$ and $98 \%$, respectively).

CONCLUSIONS: G + chemo is projected to be cost-effective versus rituximab biosimilars plus chemotherapy in the United States as firstline treatment for FL, driven by increased QALYs for G + chemo and cost savings from delayed disease progression.

Indolent non-Hodgkin lymphomas (iNHLs) constitute approximately one third of all cases of NHL, with follicular lymphoma (FL) being the most common subtype., ${ }^{1,2}$ Obinutuzumab (GA101; G) plus chemotherapy followed by obinutuzumab monotherapy $(\mathrm{G}+\mathrm{chemo})$ was shown to provide a statistically significant improvement in investigator-assessed progression-free survival (PFS) compared with rituximab plus chemotherapy followed by rituximab monotherapy $(\mathrm{R}+$ chemo) in the GALLIUM trial (NCT01332968), an open-label, multicenter, randomized phase 3 study in patients with previously untreated FL. ${ }^{3}$

In a cost-effectiveness analysis based on GALLIUM trial data and published literature, $\mathrm{G}+$ chemo was highly cost-effective versus $\mathrm{R}+$ chemo, despite its higher overall treatment cost, with an incremental cost-effectiveness ratio (ICER) of $\$ 2,300$ per quality-adjusted life-year (QALY) gained. ${ }^{4}$ This result was primarily driven by the statistically significant improvement in PFS of $\mathrm{G}+$ chemo, which increased life expectancy and reduced the cost of additional treatments and lower quality of life among patients whose disease has progressed.

Two rituximab biosimilars, rituximab-abbs (Truxima, Teva; Ra) and rituximab-pvvr (Ruxience, Pfizer; Rp), have recently been approved by the U.S. Food and Drug Administration (FDA) for patients with FL that is previously untreated or in at least partial remission. ${ }^{5}$ The FDA defines biosimilars as biological products that are highly similar to and have no clinically meaningful differences from existing FDA-approved reference products. ${ }^{6}$ The U.S. Congress created an abbreviated licensure pathway for biosimilars through the Biologics Price Competition and Innovation Act of 2009, with the intent to provide increased access to lifesaving medications at lower health care costs by encouraging competition among pharmaceutical manufacturers. ${ }^{7}$

Manufacturers are required to demonstrate that a proposed biosimilar product has no clinically meaningful differences from the reference product in terms of safety, purity, and potency (safety and effectiveness). ${ }^{6}$ Therefore, the introduction of Ra and $\mathrm{Rp}$ raises the question of whether a cheaper treatment option with the same efficacy and safety profile as $\mathrm{R}+$ chemo would influence the relative value of $\mathrm{G}+$ chemo in this patient population. The objective of this study was to estimate the U.S.-based cost-effectiveness of $\mathrm{G}+$ chemo versus $\mathrm{Ra}+$ chemo or $\mathrm{Rp}+$ chemo in a previously untreated FL population by analyzing GALLIUM trial data, average sales prices for injectable drugs, Medicare reimbursement data, and published literature.

\section{Methods}

\section{APPROACH}

We adapted a previous decision-analytic model evaluating first-line treatment with $G+$ chemo versus $R+$ chemo in patients with previously untreated FL, which was based directly on the findings of the GALLIUM trial. ${ }^{4}$ Following a review of rituximab, Ra, and Rp prescribing information, we assumed that rituximab biosimilars plus chemotherapy have the same clinical efficacy and safety profiles as $\mathrm{R}+$ chemo. All drug and administration costs were updated to current (2020) values. We also utilized updated efficacy, safety, and quality-of-life trial data. The model was used to simulate individuals' health care actions, clinical events, and health care costs over a lifetime. We used a U.S. health care sector perspective (i.e., focused on direct medical care costs only) and used a lifetime horizon. We discounted all cost and health outcomes by $3 \%$ per year. ${ }^{8}$ The model was developed in Microsoft Excel (Microsoft, Redmond, WA).

\section{MODEL STRUCTURE}

We used a 3-state Markov model framework, which included PFS, progressed disease (PD), and death (Figure 1). Health state-specific costs and utilities were applied in each monthly model cycle. Hypothetical patients entered the model in the PFS health state, where they received either $\mathrm{G}+$ chemo or rituximab biosimilar plus chemotherapy. Patients in both arms could discontinue first-line treatment 


\section{FIGURE 1 Decision Model: 3 States}

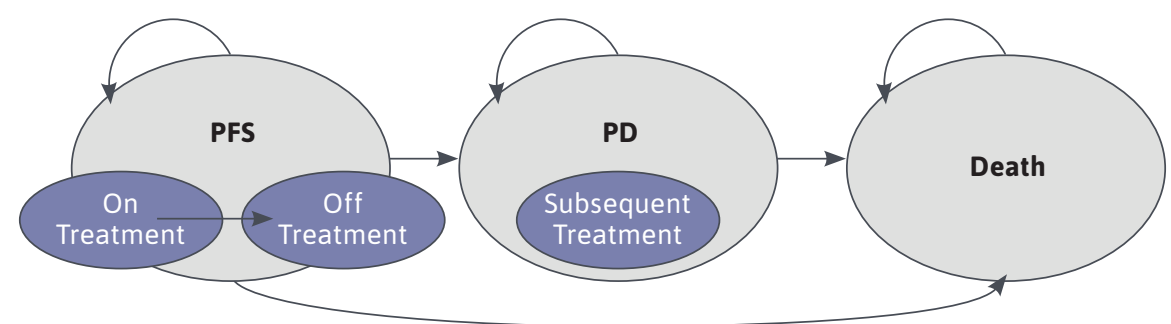

Note: All patients start in the progression-free health state, where they either receive study drugs or not (on/off treatment) during each approximate 1-month cycle. Patients can subsequently move either to another health state or stay in the same state at the end of each subsequent analysis cycle. The possible transitions are indicated by arrows; patients were simulated until death.

$P F S=$ progression-free survival; $P D=$ progressed disease . (on/off treatment) before PD during each cycle. Patients in the PFS health state who survived but whose disease progressed entered the PD state, where they remained until they died.

\section{GALLIUM CLINICAL TRIAL}

GALLIUM trial treatment regimens have been previously described. ${ }^{3,4}$ In brief, the study included patients with histologically documented, CD20-positive, previously untreated grades 1-3a advanced FL or chemotherapy-naive marginal zone lymphoma. ${ }^{3}$ GALLIUM was conducted in accordance with the Declaration of Helsinki, Good Clinical Practice guidelines, and all applicable local laws and regulations. The study protocol, its amendments, and other studyrelated materials were approved by the institutional review boards/ethics committees at all participating centers. Written informed consent was provided by all patients.

A planned analysis in 2018, after a median follow-up of 57.4 months, showed that $\mathrm{G}+$ chemo resulted in clinically meaningful improvement in the primary outcome of investigator-assessed PFS compared with $\mathrm{R}+$ chemo, with an estimated hazard ratio $(\mathrm{HR})=0.73,95 \% \mathrm{CI}=0.59-0.90$;
$\mathrm{P}=0.0034 .{ }^{9}$ Overall survival (OS) data were not statistically significant between $\mathrm{G}+$ chemo and $\mathrm{R}+$ chemo treatment arms $\quad(\mathrm{HR}=0.88, \quad 95 \%$ $\mathrm{CI}=0.61-1.27 ; \mathrm{P}=0.49$ ), as FL is an indolent disease and the mortality over the follow-up period was relatively low $(9.1 \%$ and $10.2 \%$ of $\mathrm{G}+$ chemo and $\mathrm{R}+$ chemo patients, respectively). ${ }^{9}$

\section{PRESCRIBING INFORMATION COMPARISON}

We assumed that $\mathrm{Ra}$ and $\mathrm{Rp}$, the 2 rituximab biosimilars, have the same clinical efficacy and safety as rituximab. We evaluated this with a review and comparison of obinutuzumab, rituximab, Ra, and Rp prescribing information, looking specifically at clinical trial data as well as administration methods and dosage. Both rituximab biosimilar therapies generally referenced the same clinical studies, had the same administration method (intravenous infusion), and reported similar adverse event rates. Rituximab, Ra, and Rp all listed the same dosage recommendations for FL $\left(375 \mathrm{mg} / \mathrm{m}^{2}\right){ }^{10-13}$

\section{PROGRESSION-FREE SURVIVAL MODELING}

The PFS data from the GALLIUM trial were utilized to model the probability over time of remaining in the PFS health state (Figure 1). We used parametric curves fitted to Kaplan-Meier data from GALLIUM to extrapolate survival beyond the available observation period (Supplementary Figure 1, available in online article); exponential, Weibull, log-logistic, log-normal, generalized gamma, and Gompertz functions were estimated. We selected the exponential function as the PFS curve base-case for both treatment arms due to (a) Akaike Information Criterion and Bayesian Information Criterion goodness of fit, (b) the appearance of proportional hazards (the progression hazard in $\mathrm{G}+$ chemo was a constant proportion of the progression hazard in $\mathrm{R}+$ chemo according to log-cumulative hazards plots), and (c) visual inspection.

At the time of the February 2018 data cutoff, a sustained and clinically meaningful improvement in investigator-assessed PFS was observed for $\mathrm{G}$-chemo relative to $\mathrm{R}$-chemo. The proportional hazards assumption was satisfactory to model the GALLIUM PFS curves, although the long-term duration of the treatment effect as estimated by the GALLIUM PFS HR was uncertain based on the available follow-up. Long-term follow-up data from the phase 3 PRIMA study inform this assumption; the KaplanMeier curves show that separation in PFS is maintained for rituximab versus observation up to 10 years..$^{14}$ Therefore, we assumed that the PFS treatment effect for $\mathrm{G}+$ chemo was maintained for 9 years; after 9 years, we assumed that $\mathrm{G}+$ chemo PFS was equivalent to that of $\mathrm{R}+$ chemo PFS. 


\begin{tabular}{|c|c|c|c|}
\hline Setting & Value & Varied by & Source \\
\hline \multicolumn{4}{|l|}{ General } \\
\hline Time horizon, years & 50 & Fixed & \\
\hline Cycle length, months & 1 & Fixed & \\
\hline Discount: costs, \% & 3.00 & Fixed & Neumann et al. $2016^{8}$ \\
\hline Discount QALYs, \% & 3.00 & Fixed & Neumann et al. $2016^{8}$ \\
\hline Starting age, years & 57.9 & Fixed & GALLIUM 3,9 \\
\hline \multicolumn{4}{|l|}{ Utilities } \\
\hline PFS off treatment: induction & 0.775 & Beta & GALLIUM 3,9 \\
\hline PFS off treatment: maintenance and follow-up & 0.823 & Beta & GALLIUM $^{3,9}$ \\
\hline PFS on treatment: induction & 0.825 & Beta & GALLIUM ${ }^{3,9}$ \\
\hline PFS on treatment: maintenance and follow-up & 0.836 & Beta & GALLIUM ${ }^{3,9}$ \\
\hline PD, early progression & 0.618 & Beta & Wild et al. $2006^{17}$ \\
\hline PD, late progression & 0.618 & Beta & Wild et al. $2006^{17}$ \\
\hline \multicolumn{4}{|l|}{ FS modeling } \\
\hline Distribution & Exponential & Scenario analysis & \\
\hline G+chemo-lambda & 0.0059 & Covariance matrix & \\
\hline R+chemo-lambda & 0.0084 & Covariance matrix & \\
\hline Probability of dying in PFS health state & 0.096 & Beta & \\
\hline \multicolumn{4}{|l|}{ PSS modeling } \\
\hline Distribution & Exponential & Fixed & \\
\hline Lambda & 0.0142 & Covariance matrix & \\
\hline
\end{tabular}

\section{PROGRESSION AND OVERALL SURVIVAL MODELING}

Markov model transitions out of the PFS health state were based on (a) the probability of any event (calculated as 1 minus PFS per model cycle) and (b) the probability of dying while in the PFS health state (calculated as the maximum of either the pooled GALLIUM PFS death events per month at risk or background mortality). Transitions to the PD health state were calculated as the remainder of (a) and (b). Once in the PD health state, the GALLIUM trial's pooled postprogression survival (PPS) was used to model transitions to death. We modeled an exponential function for PPS to account for the possibility that patients could die at any time in the model, that is, we assumed all patients had the same probability of death once they entered the PD health state, regardless of when progression occurred.

\section{TREATMENT}

While in PFS, patients could be on or off treatment as depicted in Figure 1. Patients were on treatment until discontinuation due to progression, toxicity, or death. We used the GALLIUM trial's time to treatment discontinuation Kaplan-Meier curves to model the proportion of patients still on treatment each week. We utilized the actual average dose per cycle (average amount per population), wherein the vial utilization is calculated based on the observed number of administrations and the mean amount of drug administered per dose. We assumed no vial sharing among patients, thus surplus drug wastage is accounted for in the model.

\section{ADVERSE EVENT PARAMETERS}

We included all GALLIUM-reported grades 3-5 adverse events that occurred in at least $5 \%$ of patients in either trial arm. ${ }^{9}$ The frequency of events was multiplied by the average cost per event to derive a total adverse event cost for each regimen. Costs per adverse event were based on Medicare severity-diagnosis-related group estimates from the Centers for Medicare \& Medicaid Services. ${ }^{15}$ 


\section{TABLE 1 Model Parameters (continued)}

\begin{tabular}{|c|c|c|c|}
\hline Drugs (Small, Large Pack Sizes, mg) & $\begin{array}{c}\text { Small Vial or Pack Cost, } \\
\$\end{array}$ & $\begin{array}{c}\text { Large Vial or Pack Cost, } \\
\$\end{array}$ & Source \\
\hline Obinutuzumab (NA, 1,000) & - & $6,055.85$ & $\mathrm{ASP}^{18}$ \\
\hline Rituximab-abbs $(100,500)$ & 652.76 & $3,263.82$ & $\mathrm{ASP}^{18}$ \\
\hline Rituximab-pvvr $(100,500)$ & 696.51 & $3,482.55$ & $\mathrm{ASP}^{18}$ \\
\hline Rituximab $(100,500)$ & 880.81 & $4,404.06$ & ASP18 \\
\hline Bendamustine $(25,100)$ & 481.98 & $1,927.92$ & $\mathrm{ASP}^{18}$ \\
\hline Cyclophosphamide $(500,1,000)$ & 163.05 & 326.09 & ASP18 \\
\hline Doxorubicin $(10,50)$ & 2.77 & 13.83 & ASP 18 \\
\hline Vincristine $(1,2)$ & 4.97 & 9.94 & $\mathrm{ASP}^{18}$ \\
\hline Prednisone (NA, 5) & - & 0.11 & $\mathrm{ASP}^{18}$ \\
\hline Adverse Events & $\begin{array}{l}\text { Value (G + chemo, } \\
\text { Rbio + chemo), \% }\end{array}$ & Varied by & Source \\
\hline Infusion-related reactions & $12.3,7.4$ & Beta & GALLIUM $^{9}$ \\
\hline Neutropenia & $46.6,39.9$ & Beta & GALLIUM $^{9}$ \\
\hline Febrile neutropenia & $7.4,4.7$ & Beta & GALLIUM $^{9}$ \\
\hline Thrombocytopenia & $6.1,2.8$ & Beta & GALLIUM $^{9}$ \\
\hline Pneumonia & $4.7,5.4$ & Beta & GALLIUM $^{9}$ \\
\hline Infection & $22.2,18.6$ & Beta & GALLIUM $^{9}$ \\
\hline Adverse Event Costs & Value, $\$$ & Varied by & Source \\
\hline Infusion-related reactions & 5,783 & Normal & CMS MS-DRG Final Rule Table, $2020^{15}$ \\
\hline Neutropenia & 5,057 & Normal & CMS MS-DRG Final Rule Table, $2020^{15}$ \\
\hline Febrile neutropenia & 8,103 & Normal & CMS MS-DRG Final Rule Table, $2020^{15}$ \\
\hline Thrombocytopenia & 9,010 & Normal & CMS MS-DRG Final Rule Table, $2020^{15}$ \\
\hline Pneumonia & 5,313 & Normal & CMS MS-DRG Final Rule Table, $2020^{15}$ \\
\hline Infection & 7,102 & Normal & CMS MS-DRG Final Rule Table, $2020^{15}$ \\
\hline \multirow[t]{2}{*}{ Cost of Progression } & Value, \$ & Varied by & \begin{tabular}{|l} 
Source \\
\end{tabular} \\
\hline & 98,622 & Normal & ASP $^{18} ;$ National LymphoCare Study $y^{20,21}$ \\
\hline $\begin{array}{l}\text { ASP = average sale price; } C M S M S-D R G=\text { Centers } \\
+ \text { +chemotherapy; } N A=\text { not applicable; } P D=\text { progre } \\
\text { Rbio + chemo = rituximab biosimilar + chemothera }\end{array}$ & $\begin{array}{l}\text { care and Medicaid Services } N \\
\text { ease; } P F S=\text { progression-free }\end{array}$ & $\begin{array}{l}\text { 1edicare Severity Diagnosis } R \\
\text { urvival; } P P S=\text { postprogression }\end{array}$ & $\begin{array}{l}\text { ated Groups; } G \text { + chemo=obinutuzumab } \\
\text { urvival; QALY = quality-adjusted life-year; }\end{array}$ \\
\hline
\end{tabular}

\section{QUALITY-OF-LIFE PARAMETERS}

EuroQoL 5D (EQ-5D) data were collected during the original treatment and follow-up period from the GALLIUM trial and were used as the primary source for informing PFS utilities in the model. The EQ-5D scores accounted for underlying disease status, treatment, and adverse events. The EQ-5D summary scores were collected at baseline, at the third cycle of induction treatment, at the end of induction visit, and at 1-year intervals thereafter until the end of the 5-year follow-up. EQ-5D scores were also collected at the first assessment after progression. We used pooled utility values for both treatment arms.

Postprogression utility data from GALLIUM were limited because responses were collected only once after progression and so did not capture advanced stages of progression..$^{16,17}$ Therefore, for the PD health state, we utilized the most widely cited utility values in the health economics literature (Table 1). ${ }^{16,17} \mathrm{~A}$ total of 222 patients with FL from 8 sites across the United Kingdom who completed the EQ-5D questionnaire were included in the utility elicitation study of Wild et al. (2006). ${ }^{17}$ Adverse event-related 


\begin{tabular}{|c|c|c|c|c|c|c|c|}
\hline \multirow[b]{2}{*}{ Regimen } & \multirow[b]{2}{*}{ Total Costs, \$ } & \multirow[b]{2}{*}{ Total Life-Years } & \multirow[b]{2}{*}{ Total QALYs } & \multicolumn{3}{|c|}{ Incremental Change for $\mathrm{G}+$ chemo } & \multirow[b]{2}{*}{ ICER (\$/QALY) } \\
\hline & & & & Cost, $\$$ & Life-Years & QALYs & \\
\hline G + chemo $(95 \%$ CR) & $\begin{array}{c}191,317 \\
(171,778-218,533)\end{array}$ & $\begin{array}{c}11.97 \\
(11.26-12.67)\end{array}$ & $\begin{array}{c}9.28 \\
(8.61-9.94)\end{array}$ & NA & NA & NA & NA \\
\hline Ra + chemo $(95 \%$ CR) & $\begin{array}{c}164,340 \\
(143,566-194,035)\end{array}$ & $\begin{array}{c}10.96 \\
(10.15-11.75)\end{array}$ & $\begin{array}{c}8.35 \\
(7.61-9.09)\end{array}$ & $\begin{array}{c}26,978 \\
(19,781-33,119)\end{array}$ & $\begin{array}{c}1.01 \\
(0.37-1.60)\end{array}$ & $\begin{array}{c}0.93 \\
(0.36-1.46)\end{array}$ & $\begin{array}{c}28,879 \\
(14,524-86,968)\end{array}$ \\
\hline Rp + chemo (95\% CR) & $\begin{array}{c}169,755 \\
(148,919-199,497)\end{array}$ & $\begin{array}{c}10.96 \\
(10.15-11.75)\end{array}$ & $\begin{array}{c}8.35 \\
(7.61-9.09)\end{array}$ & $\begin{array}{c}21,562 \\
(14,473-28,389)\end{array}$ & $\begin{array}{c}1.01 \\
(0.37-1.60)\end{array}$ & $\begin{array}{c}0.93 \\
(0.36-1.46)\end{array}$ & $\begin{array}{c}23,082 \\
(10,861-76,500)\end{array}$ \\
\hline
\end{tabular}

$C R=$ credible range; $G+$ chemo = obinutuzumab + chemotherapy; ICER = incremental cost-effectiveness ratio; $N A=$ not applicable; $Q A L Y=$ quality-adjusted life-year; $R a+c h e m o=$ rituximab-abbs + chemotherapy; $R p+$ chemo $=$ rituximab-pvvr+chemotherapy.

disutilities were not included due to the low frequency of adverse events in both treatment arms.

\section{TREATMENT COST PARAMETERS}

We used intravenous drug average sales prices obtained from the 2020 Centers for Medicare and Medicaid Services Medicare Fee-for-Service Part B Drugs database $(\mathrm{G}=\$ 6,055.85 / 1,000 \mathrm{~mL}$ vial; $\mathrm{Ra}=\$ 3,263.82 / 500 \mathrm{~mL}$ vial and $\$ 652.76 / 100 \mathrm{~mL}$ vial; $\mathrm{Rp}=\$ 3,482.55 / 500 \mathrm{~mL}$ vial and $\$ 696.51 / 100 \mathrm{~mL}$ vial). ${ }^{18}$ The drug cost per patient per administration was calculated using average patient baseline characteristics and regimens from the relevant clinical trial publications and prescribing information. All per-vial drug costs accounted for wastage. Intravenous infusion costs per administration were determined using March 2020 Medicare reimbursement rates (Table 1). ${ }^{19}$

\section{POSTPROGRESSION TREATMENT COSTS}

Salvage therapy costs following progression were derived as a weighted cost of an estimated mix of salvage therapies based on postprogression market share estimates (data on file, Genentech). We assumed that approximately $69 \%$ of patients who progress will go on to receive second-line treatment and beyond, based on results from the National LymphoCare study. ${ }^{20,21}$ We assumed that salvage therapy costs were equivalent for $\mathrm{G}+$ chemo and rituximab biosimilars plus chemotherapy patients in the model, and we assessed it as a 1-time cost of $\$ 98,622$, based on the above assumptions upon entrance into the PD health state. All rituximab-based regimens in the salvage therapy mix were assumed to substitute the average cost of rituximab biosimilars for rituximab.

\section{ANALYSIS}

We calculated lifetime direct medical costs, QALYs, and life-years for the $\mathrm{G}+$ chemo, $\mathrm{Ra}+$ chemo, and $\mathrm{Rp}+$ chemo regimens in an untreated FL population. The ICER was calculated as the difference $(\Delta)$ in costs divided by the difference in QALYs. We performed 1-way and probabilistic sensitivity analyses to assess how uncertainty in model parameter estimates affected the results. In 1-way sensitivity analysis, 1 parameter at a time is varied to its low and high value while keeping all parameters constant. In probabilistic sensitivity analysis, all model parameters were simultaneously, randomly varied according to an assigned probability distribution over 5,000 simulations. Ninety-five percent credible ranges (95\% CRs) were calculated for each model result.

We utilized scenario analyses of $\mathrm{G}+$ chemo versus $\mathrm{Rp}+$ chemo (the less-expensive comparator) to evaluate important assumptions in our model, including variation of the PFS treatment effect duration (9 years in base-case analysis) and variation of our parametric survival curve selections. We also modeled a scenario with differences in postprogression mortality for patients who progress within the first 2 years versus those who progress later. In patients with FL who received first-line rituximab plus cyclophosphamide, doxorubicin, vincristine, and prednisone (R-CHOP), early progression has been associated with poor survival outcomes. ${ }^{21,22}$ We used the GALLIUM trial PPS for early progressors and PRIMA study PPS for late progressors. Finally, we performed a scenario analysis to compare this analysis with our previous cost-effectiveness model of $\mathrm{G}+$ chemo versus $\mathrm{R}+$ chemo by substituting rituximab cost for rituximab biosimilar costs.

\section{Results}

$\mathrm{G}+$ chemo resulted in 9.28 QALYs (95\% $\mathrm{CR}=8.61-9.94)$ and 11.97 (95\% CR=11.26-12.67) life-years gained, whereas $\mathrm{Ra}+$ chemo and $\mathrm{Rp}+$ chemo resulted in fewer QALYs $(8.35$, 95\% $\mathrm{CR}=7.61-9.09)$ and life-years $(10.96,95 \% \mathrm{CR}=10.15-11.75$; 


\section{FIGURE 2 Costs Incorporated in the Model}

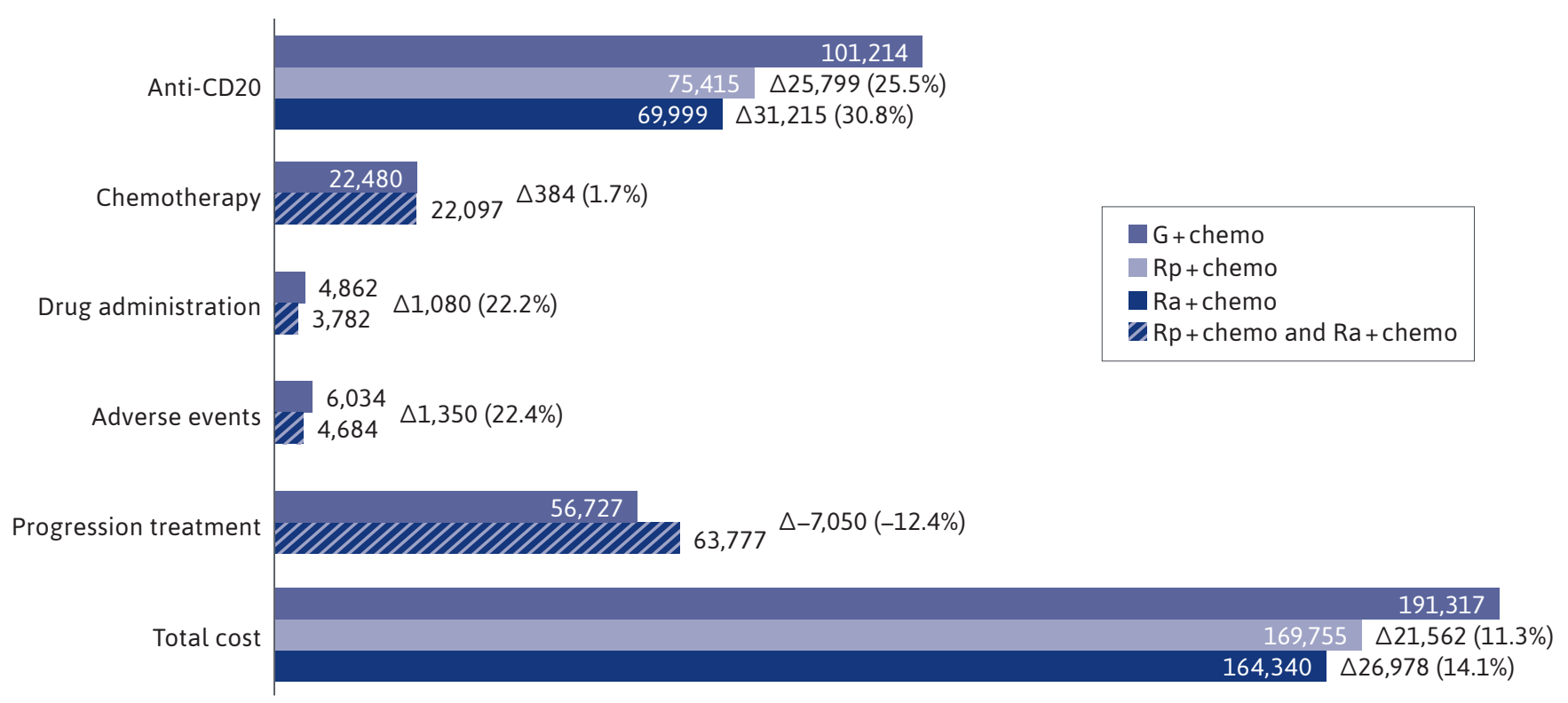

Note: Costs are in U.S. dollars.

$\Delta=$ change; $G+$ chemo=obinutuzumab +chemotherapy; $R a+$ chemo=rituximab-abbs +chemotherapy; $R p+c h e m o=$ rituximab-pvvr + chemotherapy.

Table 2). The incremental difference in QALYs was 0.93 (95\% $\mathrm{CR}=0.36-1.46)$. The incremental QALYs gained for $\mathrm{G}+$ chemo predicted by the model were primarily driven by the difference in modeled PFS curves.

Total costs of care were $\$ 191,317 \quad(95 \% \quad C R=\$ 171,778-$ $\$ 218,533)$ for $\mathrm{G}+$ chemo versus $\$ 164,340(\Delta 14.1 \% ; 95 \%$ $\mathrm{CR}=\$ 143,566-\$ 194,035)$ for $\mathrm{Ra}+$ chemo and $\$ 169,755(\Delta 11.3 \%$; 95\% $\mathrm{CR}=\$ 148,919-\$ 199,497$ ) for $\mathrm{Rp}+$ chemo (Table 2 and Figure 2). Average total drug and administration costs were $\$ 128,557$ for $\mathrm{G}+$ chemo, $\$ 95,878(\Delta 25.4 \%)$ for $\mathrm{Ra}+$ chemo, and $\$ 101,294(\triangle 21.2 \%)$ for $\mathrm{Rp}+\mathrm{chemo}$; the incremental total drug and administration cost differences were $\$ 32,678$ and $\$ 27,263$, respectively.

Average adverse event costs were higher for $\mathrm{G}+$ chemo $(\$ 6,034)$ versus $\mathrm{Ra}+$ chemo and $\mathrm{Rp}+$ chemo $(\$ 4,684)$ and were driven across all treatments predominantly by costs associated with neutropenia and infection. There were cost savings of $\$ 7,050(\Delta-12.4 \% ; 95 \% \quad C R=-\$ 12,821$ to $-\$ 2,542)$ related to disease progression for $\mathrm{G}+$ chemo $(\$ 56,727)$ compared with $\mathrm{Ra}+$ chemo and $\mathrm{Rp}+$ chemo $(\$ 63,777)$. The ICER for $\mathrm{G}+$ chemo compared with $\mathrm{Ra}+$ chemo was $\$ 28,879$ per QALY gained (95\% CR=\$14,524-\$86,968), and the ICER for $\mathrm{G}+$ chemo compared with $\mathrm{Rp}+$ chemo was $\$ 23,082$ per QALY gained (95\% CR $=\$ 10,861-\$ 76,500)$.
One-way sensitivity analysis for $\mathrm{G}+$ chemo versus $\mathrm{Ra}+$ chemo or $\mathrm{Rp}+$ chemo showed that our results were most sensitive to the cost of obinutuzumab, the $\mathrm{G}+$ chemo PFS median survival, and the cost of the rituximab biosimilar. ICERs were generally robust to changes in model parameters, staying below $\$ 50,000$ per QALY gained in all 1-way analyses for both the $\mathrm{Ra}+$ chemo comparison and the $\mathrm{Rp}+$ chemo comparison except for the low value for $\mathrm{G}+$ chemo PFS median survival and the high value for obinutuzumab (Figure 3).

Probabilistic sensitivity analysis results are presented as a cost-effectiveness acceptability curve, which shows the Bayesian probability that the results are cost-effective at increasing willingness to pay per QALY thresholds (Supplementary Figure 2, available in online article). In these analyses, $\mathrm{G}+$ chemo was cost-effective at the $\$ 50,000$ and $\$ 100,000$ per QALY thresholds versus both $\mathrm{Ra}+$ chemo (88\% and 98\% probabilities of cost-effectiveness, respectively) and $\mathrm{Rp}+$ chemo (93\% and 98\%, respectively).

Varying the parametric distributions used for the PFS curves resulted in ICERs ranging from $\$ 19,522$ to $\$ 39,555$ per QALY gained when comparing $\mathrm{G}+$ chemo with $\mathrm{Ra}+$ chemo (Supplementary Table 1, available in online article). The 


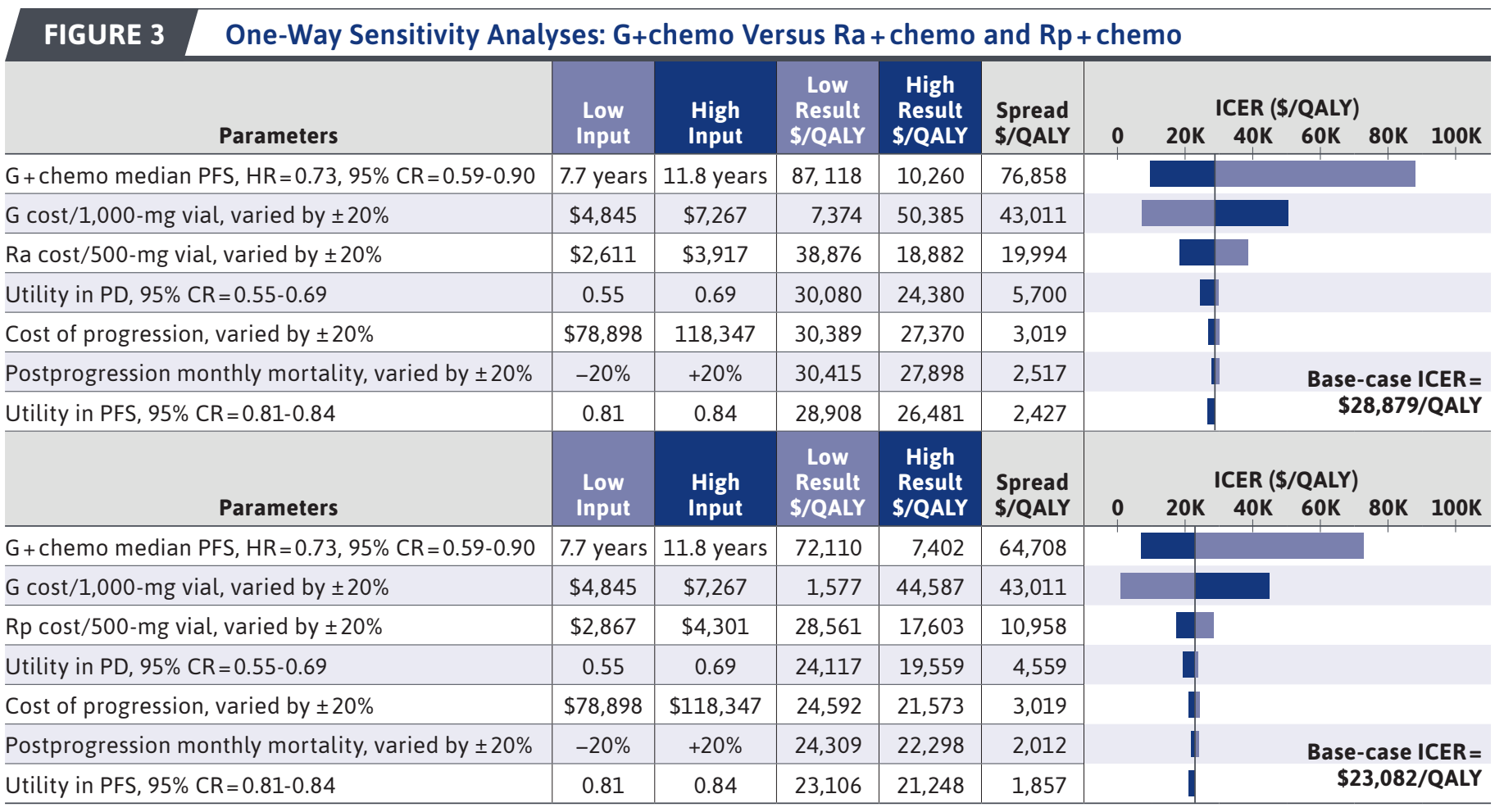

$C R=$ credible range; $G=$ obinutuzumab; $G+$ chemo = obinutuzumab + chemotherapy; $H R=$ hazard ratio; $I C E R=$ incremental cost-effectiveness ratio; $P D=$ progressed disease; PFS = progression-free survival; $Q A L Y=$ quality-adjusted life-year; $R a=$ rituximab-abbs; $R p=$ rituximab pvvr.

scenario in which we used PRIMA data to model a mortality difference in early versus late progressors increased OS for both comparators; however incremental QALYs increased only slightly and had little effect on the ICER. Reduction in the cutoff time for the treatment effect had a more notable effect on model results. For example, when we decreased the cutoff for treatment effect from 9 to 5 years (the length of current follow-up), the ICER increased to $\$ 46,829$ per QALY gained. These results were driven by the differences in patients who progressed; the shorter the PFS treatment effect was modeled, the less time patients in the $\mathrm{G}+$ chemo arm remained in the PFS health state with higher quality of life and without costs of PD. Substituting rituximab for rituximab biosimilars decreased the incremental cost of $\mathrm{G}+$ chemo, and the ICER decreased to $\$ 2,385$ per QALY gained.

\section{Discussion}

Using a model based on findings from the GALLIUM trial, we estimated the cost-effectiveness of $\mathrm{G}+$ chemo versus $\mathrm{Ra}+$ chemo and $\mathrm{Rp}+$ chemo in previously untreated FL patients. Our findings expand on the previously performed cost-utility analysis of $\mathrm{G}+$ chemo versus $\mathrm{R}+$ chemo and highlight the key health economic implications for stakeholders facing treatment, recommendation, or coverage and reimbursement decisions relating to previously untreated FL. $\mathrm{G}+$ chemo was estimated to increase both time in PFS and OS compared with $\mathrm{Ra}+$ chemo and $\mathrm{Rp}+$ chemo. Despite the lower overall cost of rituximab biosimilars plus chemotherapy compared with $\mathrm{G}+$ chemo, the ICERs for $\mathrm{G}+$ chemo versus rituximab biosimilar-based regimens fall well within commonly accepted thresholds for cost-effectiveness in the United States. . $32,24^{2}$

Our results from the model indicate that $\mathrm{G}+$ chemo continues to offer good economic value, including improved patient outcomes, compared with $\mathrm{R}+$ chemo or equivalents based on rituximab biosimilars, and that value-based policy decisions should consider $\mathrm{G}+$ chemo as standard of care for patients with untreated FL. Several factors drive the value of $\mathrm{G}+$ chemo in our economic model. First, there was a significant and clinically meaningful difference in PFS in the GALLIUM trial, which confers additional QALYs on $\mathrm{G}+$ chemo. Second, the $\mathrm{G}+$ chemo incremental total 
costs versus $\mathrm{Ra}+$ chemo $(\$ 26,978)$ and $\mathrm{Rp}+$ chemo $(\$ 21,562)$ were not high enough to drive the ICER beyond established willingness to pay per QALY thresholds. Third, we estimated cost savings of $\$ 7,050$ from delaying disease progression. Fourth, although the difference in early (3-year) progression between $\mathrm{G}+$ chemo and $\mathrm{R}+$ chemo was approximately $80 \%$ versus $73 \%$, respectively, ${ }^{25}$ our comprehensive investigation of variation in parametric functions, parameters, as well as the analysis to accommodate the timing of progression, indicate the robustness of our results.

\section{LIMITATIONS}

Our analysis had several limitations. First, our projections for PFS are uncertain given the available follow-up data from GALLIUM. We addressed this by evaluating a number of different parametric functions and exploring the external validity of the modeled curves versus the survival observed in the PRIMA trial. The clinically meaningful difference in PFS with $\mathrm{G}+$ chemo versus $\mathrm{R}+$ chemo and estimated savings with postprogression costs, however, support the conclusion that $\mathrm{G}+$ chemo appears to offer good value for money.

Second, OS data at the time of our analysis were based on 57.4 months' median observation time. Nonetheless, our results demonstrated that $\mathrm{G}+$ chemo remained cost-effective versus $\mathrm{Ra}+$ chemo and $\mathrm{Rp}+$ chemo across a range of modeled survival approaches based on pooled PPS curves from GALLIUM.

Third, our assumption of a common cost of progression for all patients who progress reflected market share estimates of second-line treatment regardless of first-line therapy. We varied this cost by $\pm 20 \%$ to account for a wide range of possible progression costs, and our ICER remained below a \$50,000 willingness-to-pay threshold.

Finally, we note that drug efficacy and safety may be more favorable in clinical trials than in real-world clinical care, thus real-world outcomes may differ from those considered in the model.

\section{Conclusions}

Our U.S.-based analysis suggests that treatment with $\mathrm{G}+$ chemo is likely cost-effective compared with regimens based on rituximab biosimilars plus chemotherapy in patients with previously untreated FL. This cost-effectiveness is driven by an improvement in modeled life expectancy and quality of life as well as delaying treatments for progression. The results were robust to multiple sensitivity analyses. This economic analysis provides policymakers with a framework for evaluating the clinical and economic value of obinutuzumab in this clinical setting in the context of rituximab biosimilars in the United States.

\section{DATA-SHARING STATEMENT}

Qualified researchers may request access to individual patient-level data through the clinical study data request platform (https://vivli.org/). Further details on Roche's criteria for eligible studies are available here: https://vivli.org/members/ourmembers/. For further details on Roche's Global Policy on the Sharing of Clinical Information and how to request access to related clinical study documents, see here: https://www.roche. com/research_and_development/who_ we_are_how_we_work/clinical_trials/ our commitment to data sharing.htm.

\section{DISCLOSURES}

This study was funded by Genentech, a member of the Roche Group. The study sponsor was involved in study design, data interpretation, and writing of the report. All authors approved the decision to submit the report for publication.
Spencer and Guzauskas report fees from Genentech during the conduct of the study. Felizzi was employed by F. Hoffmann-La Roche at the time this study was conducted; Launonen is an employees of F. Hoffmann-La Roche. Felizzi and Launonen previously had share ownership in Novartis. Dawson and Masaquel are employees of Genentech, and they have stock options in F. Hoffmann-La Roche. Veenstra reports fees from Genentech, during the conduct of this study and outside of the submitted work.

This work was presented, in part, at the AACR Virtual Meeting Advances in Malignant Lymphoma meeting (virtual; August 17-19, 2020) and the SOHO annual meeting (virtual; September 9-12, 2020).

\section{ACKNOWLEDGMENTS}

The GALLIUM trial was sponsored by F. Hoffmann-La Roche. Third-party editorial assistance, under the guidance of the authors, was provided by Katie Smith of Ashfield MedComms, an Ashfield Health company, and was funded by F. Hoffmann-La Roche.

\section{REFERENCES}

1. Campo E, Swerdlow SH, Harris NL, Pileri S, Stein H, Jaffe ES. The 2008 WHO classification of lymphoid neoplasms and beyond: evolving concepts and practical applications. Blood. 2011;117(19):5019-32.

2. National Comprehensive Cancer Network. NCCN clinical practice guidelines in oncology (NCCN Guidelines): B-cell lymphomas. Version 1.2021. January 20, 2021. Accessed January 22, 2021. https://www.nccn.org/professionals/ physician gls/pdf/b-cell.pdf

3. Marcus R, Davies A, Ando K, et al. Obinutuzumab for the first-line treatment of follicular lymphoma. N Engl J Med. 2017;377(14):1331-44.

4. Guzauskas GF, Masaquel A, Thuresson PO, Dawson K, Veenstra DL. Obinutuzumab plus chemotherapy followed by obinutuzumab monotherapy is cost-effective vs. rituximab plus chemotherapy followed by rituximab monotherapy for previously untreated follicular lymphoma patients in the United States. Leuk Lymphoma. 2019;60(7):1668-76. 
5. U.S. Food and Drug Administration. FDA approves first biosimilar for treatment of adult patients with nonHodgkin's lymphoma [press release]. November 28, 2018. Accessed January 22, 2021. https://www.fda.gov/ news-events/press-announcements/ fda-approves-first-biosimilar-treatmentadult-patients-non-hodgkins-lymphoma

6. U.S. Food and Drug Administration. Biosimilar and interchangeable products. October 23, 2017. Accessed January 22, 2021. https:// www.fda.gov/drugs/biosimilars/ biosimilar-and-interchangeable-products

7. U.S. Food and Drug Administration. Biologics Price Competition and Innovation Act of 2009. HR 3590-686-703. 2009. Accessed January 22, 2021. https:// www.fda.gov/media/78946/download

8. Neumann PJ, Sanders GD, Russell LB, Siegel JE, Ganiats TG. Cost-Effectiveness in Health and Medicine. New York: Oxford University Press; 2016.

9. Townsend W, Buske C, Cartron G, et al. Obinutuzumab-based immunochemotherapy prolongs progression-free survival and time to next anti-lymphoma treatment in patients with previously untreated follicular lymphoma: four-year results from the Phase III GALLIUM study. Blood. 2018;132(Supplement 1):1597.

10. Gazyva (obinutuzumab) injection, for intravenous use. Genentech. March 2020. Accessed January 22, 2021. https://www. gene.com/download/pdf/gazyva_prescribing.pdf

11. Truxima (rituximab-abbs) injection, for intravenous use. Teva. May 2020. Accessed January 22, 2021. https:// www.truximahcp.com/globalassets/ truxima-dtc/pdfs/truxima-prescribinginformation.pdf
12. Rituxan (rituximab) injection, for intravenous use. Genentech. August 2020. Accessed January 22, 2021. https://www. gene.com/download/pdf/rituxan_prescribing.pdf

13. Ruxience (rituximab-pvvr) injection, for intravenous use. Pfizer. May 2020. Accessed January 22, 2021. http:// labeling.pfizer.com/ShowLabeling. aspx?id=12090

14. Bachy E, Seymour JF, Feugier P, et al. Sustained progression-free survival benefit of rituximab maintenance in patients with follicular lymphoma: longterm results of the PRIMA study. J Clin Oncol. 2019;37(31):2815-24.

15. Centers for Medicare \& Medicaid Services. FY 2020 final rule and correction notice tables. Accessed January 22, 2021. https://www.cms.gov/Medicare/ Medicare-Fee-for-Service-Payment/ AcuteInpatientPPS/FY2020-IPPSFinal-Rule-Home-Page-Items/ FY2020-IPPS-Final-Rule-Tables.html

16. Pettengell R, Donatti C, Hoskin P, et al. The impact of follicular lymphoma on health-related quality of life. Ann Oncol. 2008;19(3):570-76.

17. Wild D, Walker M, Pettengell R, Lewis G. PCN62 utility elicitation in patients with follicular lymphoma. Value Health. 2006;9(6):A294.

18. Centers for Medicare and Medicaid Services. 2020 ASP drug pricing files (July). Accessed February 3, 2021. https://www.cms.gov/medicare/ medicare-part-b-drug-average-salesprice/2020-asp-drug-pricing-files
19. Centers for Medicare and Medicaid Services. Physician Fee Schedule Search. Accessed January 22, 2021. www.cms.gov/ apps/physician-fee-schedule/licenseagreement.aspx

20. Link BK, Day BM, Zhou X, et al. Second-line and subsequent therapy and outcomes for follicular lymphoma in the United States: data from the observational National LymphoCare Study. Br J Haematol. 2019;184(4):660-63.

21. Casulo C, Byrtek M, Dawson KL, et al. Early relapse of follicular lymphoma after rituximab plus cyclophosphamide, doxorubicin, vincristine, and prednisone defines patients at high risk for death: an analysis from the National LymphoCare Study. J Clin Oncol. 2015;33(23):2516-22.

22. Maurer MJ, Bachy E, Ghesquieres H, et al. Early event status informs subsequent outcome in newly diagnosed follicular lymphoma. Am J Hematol. 2016;91(11):1096-101.

23. Monga N, Garside J, Gurung B, et al. Cost-effectiveness analyses, costs and resource use, and health-related quality of life in patients with follicular or marginal zone lymphoma: systematic reviews. Pharmacoecon Open. 2020;4(4):575-91.

24. Neumann PJ, Cohen JT, Weinstein MC. Updating cost-effectiveness-the curious resilience of the $\$ 50,000$-perQALY threshold. N Engl J Med. 2014;371(9):796-97.

25. Nair R, Tabchi S, Hagermeister F. Obinutuzumab treatment of follicular lymphoma. N Engl J Med. 2017;377(26):2605-06. 